\title{
Ramucirumab plus paclitaxel or FOLFIRI in platinum-refractory advanced or metastatic gastric or gastroesophageal junction adenocarcinoma-experience at two centres
}

\author{
Ursula M. Vogl ${ }^{1}$, Laurenz Vormittag ${ }^{2}$, Thomas Winkler ${ }^{2}$, Alice Kafka ${ }^{2}$, Olivia Weiser-Jasch ${ }^{2}$, \\ Bettina Heinrich ${ }^{2}$, Sophie Roider-Schur ${ }^{2}$, Haleh Andalibi ${ }^{1}$, Eva Autzinger ${ }^{1}$, Wolfgang Schima ${ }^{3}$, \\ Alexander Klaus ${ }^{4}$, Johannes Zacherl ${ }^{5}$, Günter Michael Wimberger ${ }^{6}$, Leopold Öhler ${ }^{1,2}$
}

${ }^{1}$ Department of Medicine I, Oncology, Barmherzige Schwestern Krankenhaus Wien, Vienna, Austria; ${ }^{2}$ Department of Medicine I, Oncology, St. Josef Krankenhaus, Vienna, Austria; ${ }^{3}$ Department of Diagnostic and Interventional Radiology, Barmherzige Schwestern Krankenhaus Wien, St. Josef Krankenhaus and Krankenhaus Göttlicher Heiland, Vienna, Austria; ${ }^{4}$ Department of Surgery, Barmherzige Schwestern Krankenhaus Wien, Vienna, Austria; ${ }^{5}$ Department of Surgery, St. Josef Krankenhaus, Vienna, Austria; ${ }^{6}$ Department of Surgery, Krankenhaus Göttlicher Heiland, Vienna, Austria Contributions: (I) Conception and design: UM Vogl, L Öhler; (II) Administratice support: None; (III) Provisions of study material or patients: None; (IV) Collection and assembly of data: All authors; (V) Data analysis and interpretation: All authors; (VI) Manuscript writing: All authors; (VII) Final approval of manuscript: All authors.

Correspondence to: Leopold Öhler, MD. Department of Medicine I, Oncology Barmherzige Schwestern Krankenhaus Wien, Vienna, Austria; Department of Internal Medicine I, Oncology, St. Josef Krankenhaus, Vienna, Austria. Email: leopold.oehler@bhs.at.

Background: Ramucirumab is a VEGFR-2 antibody that has proven to prolong overall survival (OS) in patients with pretreated metastatic gastric/gastrooesophageal junction (GEJ) adenocarcinoma. We present data from patients treated with ramucirumab and paclitaxel or FOLFIRI after failure of at least one platinum- and 5-FU-containing chemotherapy (CHT) regimen.

Methods: In this retrospective two-center study, 56 patients with metastatic gastric cancer (47\%) or adenocarcinoma of the GEJ (53\%) were treated with paclitaxel and ramucirumab $(n=38)$ as second-line $(75 \%)$ or beyond second-line (25\%) therapy. FOLFIRI-ramucirumab (FOLFIRI-R) $(n=16)$ was given to patients with a short interval between taxane-based perioperative CHT and occurrence of metastatic disease or to those ineligible for paclitaxel.

Results: The median progression-free survival (PFS) and OS for patients treated with paclitaxelramucirumab (pacl-R) were 2.9 (95\% CI: 2.3-3.6) and 4.4 (4.1-4.7) months, respectively, and those for patients treated with FOLFIRI-R were 5.9 (95\% CI: 0.35-11.4) and 8.3 (6.6-10) months, respectively $(\mathrm{P}=0.05)$. We observed a trend towards prolonged PFS after perioperative taxane-based FLOT CHT $(\mathrm{n}=12)$ with FOLFIRI-R compared with pacl-R. Adverse events were manageable, with neutropenia and polyneuropathy (PNP) being the most common events. More than two treatment lines were given to $48.2 \%$ of patients.

Conclusions: The use of ramucirumab in combination with FOLFIRI showed favourable PFS and OS in patients with prior treatments with platinum and/or taxane-based agents and allows further treatment lines after progression. In patients with taxane pretreatment or persistent high-grade PNP, the combination of FOLFIRI-R might be a promising combination.

Keywords: Gastric cancer; ramucirumab; paclitaxel; FOLFIRI; FLOT

Submitted Nov 29, 2019. Accepted for publication Feb 05, 2020.

doi: 10.21037/jgo.2020.03.10

View this article at: http://dx.doi.org/10.21037/jgo.2020.03.10 


\section{Introduction}

Gastric cancer is the fourth most prevalent malignancy worldwide, although the incidence has decreased in the western world $(1,2)$. This development is paralleled by a remarkable increase of adenocarcinoma of the GEJ in industrial countries (3). Despite improvements in the systemic therapy of gastric and gastroesophageal (GEC) cancer, the survival of patients with metastatic disease remains poor. Even when detected early and treated with perioperative chemotherapy (CHT), the 5 -year survival rate ranges from $36 \%$ to $45 \%(4,5)$. Research characterizing molecular biology and signalling pathways has revealed that angiogenesis is one of the main drivers of disease progression. Tumours secrete and produce vascular endothelial growth factor (VEGF) and related receptors to enhance neovascularization. Increased VEGF expression has been found in approximately $43 \%$ of gastric cancers and is associated with advanced stage, a high risk of recurrence and a poor prognosis (6). Several targeted agents and antibodies against VEGF focusing on angiogenesis have been studied in clinical trials. Bevacizumab, an antibody against soluble VEGF-A approved for several entities, has shown negative results in 4 phase III trials in the metastatic and perioperative setting (7-10). In contrast, ramucirumab, which targets VEGFR-2, has shown a significant improvement in OS as a second-line treatment $v$ s. placebo both in combination with paclitaxel [9.6 vs. 7.4 months, respectively, RAINBOW trial (11)] and as a single agent [5.2 vs. 3.8 months, respectively, REGARD trial (12)] after failure of platinum- and 5-FU-based CHT. The biological rationale for investigating an antibody targeting VEGFR-2 has been shown by Liu and colleagues (13). Gastric cancers overexpressing VEGFR-2 have a poor outcome, and therefore, VEGFR-2 might be a negative prognostic marker (13). To date, there are no biomarkers available for selecting patients who benefit most from anti-angiogenesis strategies.

Small molecules (tyrosine kinase inhibitors, TKIs), such as sunitinib, sorafenib or regorafenib, do not show activity in the treatment of metastatic gastric cancer (14-16). Only one phase III trial on the VEGF TKI apatinib was positive-but only in an Asian population (17).

First-line therapy for inoperable, locally advanced or metastatic disease is mainly driven by the status of the HER-2 receptor. Recommended first-line regimens include platinum-based CHT, which is combined with trastuzumab in the case of HER-2 overexpression. Other targeted agents have not been successful in improving the response in this setting. In a phase III trial, the addition of ramucirumab to cisplatin and 5-FU did not improve overall survival (OS) (18). Doublets are generally preferred, since triple CHT, such as DCF or ECF, has not proven a survival benefit but does enhance toxicity. Intensive treatment should be reserved only for patients in need of a rapid response. In general, an Eastern Cooperative Oncology Group (ECOG) performance status of less than or equal to 2 is recommended to evaluate patients for palliative treatment.

Since the detection rates of gastric cancers are increasing, more patients are diagnosed in locally advanced but operable stages, making them candidates for perioperative CHT. The standard regimen recommended in guidelines is the FLOT regimen, which contains docetaxel (4). Therefore, a significant percentage of patients receive a taxane-based regimen at an early stage of their disease. Despite the potential of achieving a pathologic complete response (pCR), a high number of patients relapsesome even within 6 months. In particular, the subgroup of patients with an early relapse within 6 months might have taxane-resistant disease. Moreover, residual polyneuropathy from previous treatments containing platinum-based regimens renders continued neurotoxic agents unfeasible for further treatment.

FOLFIRI and ramucirumab have demonstrated safety and activity in second-line colorectal cancer, but such data are missing in gastric cancer.

Despite the lack of data, FOLFIRI-R is used off-label in daily clinical practice, on the basis of data reported by Klempner and colleagues (19) and at this year's ASCO Annual Meeting presented abstract of the interim analysis of the RAMIRIS trial (20). Since FLOT has become the standard recommended regimen in perioperative and sometimes even palliative first-line settings for patients in need of a rapid response, a rechallenge of a taxane is not an attractive treatment choice in the palliative setting.

We assessed in this retrospective study the outcome of PFS, OS and overall response rate (ORR) from 56 patients treated with ramucirumab in combination with paclitaxel or FOLFIRI in $2 \mathrm{~L}$ or beyond 2L. Additionally we present data of a patient cohort of taxane (mostly FLOT)-pretreated patients.

\section{Methods}

Between November 2014 and January 2019, fifty-six consecutive patients were treated at our institutions for 
metastatic gastric or GEJ cancer with palliative CHT. The patients' baseline characteristics are shown in Table 1. The median age was 64 years (38-82 years), with 36 patients being male. More than half of patients $(55.4 \%)$ presented with a good performance status $(\mathrm{ECOG} \leq 1)$. Patients predominantly presented with stage IV disease (53.6\%) and poorly differentiated tumours. Diffuse infiltrating type was the most frequent histology (70\%), and $5.4 \%$ were positive for HER-2.

In the era between 2014 and 2016, the most commonly used regimen in first-line therapy was DCF or cisplatin/5FU. After new guidelines recommended deferring anthracycline use, EOX or ECF was used less commonly from 2016 onwards. If relapse occurred within 6 months after perioperative therapy, perioperative CHT was considered as a first-line palliative treatment.

Ramucirumab was administered at $8 \mathrm{mg} / \mathrm{kg}$ body weight (BW) either as monotherapy biweekly or in combination with paclitaxel or FOLFIRI. Paclitaxel was given at $80 \mathrm{mg} / \mathrm{m}^{2}$ weekly on days 1,8 , and 15 every four weeks. Ramucirumab was licensed in Austria in December 2014; therefore, a small number of patients received ramucirumab in a compassionate use programme. FOLFIRI was scheduled every two weeks $\left(5-\mathrm{FU} 200 \mathrm{mg} / \mathrm{m}^{2}\right.$ i.v. bolus application and $2,400 \mathrm{mg} / \mathrm{m}^{2}$ over 46 hours continuously, irinotecan $180 \mathrm{mg} / \mathrm{m}^{2}$ i.v. over $90 \mathrm{~min}$ ) based on published trials in colorectal cancer (RAISE phase III study) (21). One patient with ECOG 2 received FOLFIRI in a modified $80 \%$ dose. OS was measured from the start of first palliative CHT until death. The response rates were evaluated using Response Evaluation Criteria in Solid Tumours (RECIST 1.1) criteria (22). Adverse events were evaluated and graded through a review of chart documentation according to the Common Terminology Criteria for Adverse Events (CTCAE version 4.0). The median PFS and OS were determined using the Kaplan-Meier method. Statistical analysis was performed using SPSS for Windows v23 (IBM, Armonk, NY, USA). Statistical significance was indicated by $\mathrm{P}<0.05$ using log-rank and Breslow tests.

\section{Results}

A total of fifty-six consecutive patients with inoperable locally advanced or metastatic disease were included in our retrospective analysis.

Twenty-nine $(52 \%)$ of all patients had received pretreatment with perioperative CHT because of locally advanced disease $(n=26)$ or peritoneal carcinomatosis $(n=3)$ as a single metastatic site and were suitable for a curative approach with peritonectomy and intraperitoneal CHT (HIPEC).

The FLOT protocol (4) was used in $41 \%$ of perioperatively treated patients $(n=12)$, resulting in a pCR in $25 \%(\mathrm{n}=3)$.

The median time from the completion of perioperative CHT to relapse was 10.6 months (range, 0-25 months). Patients with a pCR after neoadjuvant CHT did not experience significantly longer disease-free survival than patients with residual disease $(\mathrm{P}=0.51)$.

Patients with stage IV disease at the time of diagnosis $(n=30)$ or after recurrence $(n=56)$ mostly had oligometastatic disease $(n=35)$, with peritoneal carcinomatosis $(50 \%)$ and lymph node (41\%) and liver (25\%) metastases being the most frequent.

\section{First-line palliative treatment}

First-line palliative treatment consisted of FLOT $(n=12)$ given perioperatively and counted as first-line when relapse occurred within 6 months), cisplatin or carboplatin/5-FU $(n=13)$, FOLFOX $(n=7)$ or DCF $(n=4)$ or other alternative treatment regimen $(\mathrm{n}=17)$.

Patients with HER-2-positive disease $(\mathrm{n}=3)$ all received CHT in line with the published TOGA trial data (23).

Patients received a median number of two treatment lines in the palliative setting, of which $48 \%$ were eligible for three or more treatment lines.

\section{Second-line treatment and later treatment lines containing ramucirumab}

Patients with advanced gastric (46\%) or GEJ adenocarcinoma (54\%) were treated with paclitaxel and ramucirumab $(\mathrm{n}=38)$ or ramucirumab monotherapy $(\mathrm{n}=2)$ as a second-line $(86 \%)$ or beyond second-line $(14 \%)$ therapy. FOLFIRI-R ( $\mathrm{n}=16)$ was given to patients who experienced relapse less than 6 months after taxane-based perioperative CHT or to patients with persistent polyneuropathy (PNP). Patient outcomes concerning PFS, OS and the ORR regarding the different treatment combinations are summarized in Table 2.

The median PFS and OS of all patients receiving ramucirumab and CHT were 3 (95\% CI: 2.2-3.8) and 5 (95\% CI: 2.9-7) months, respectively. There was no significant difference in outcomes depending on the treatment line $(2 \mathrm{~L}$ $v s$. $>2 \mathrm{~L}$ ). Stable disease was observed in $48.9 \%$ of patients, 
Table 1 Patients' baseline characteristics

\begin{tabular}{|c|c|c|}
\hline Variable & $\mathrm{n}$ & $\%$ \\
\hline Total number of patients & 56 & 100 \\
\hline Male & 36 & 64 \\
\hline Female & 20 & 36 \\
\hline Median age (range), years & 64 [38-82] & \\
\hline \multicolumn{3}{|l|}{ ECOG } \\
\hline 0 & 31 & 55.4 \\
\hline$>1$ & 25 & 44.6 \\
\hline \multicolumn{3}{|l|}{ Initial stage, grade } \\
\hline II, III & 26 & 46.4 \\
\hline IV & 30 & 53.6 \\
\hline G3 & 51 & 91.1 \\
\hline \multicolumn{3}{|l|}{ Histology } \\
\hline Diffuse infiltrating type & 39 & 69.6 \\
\hline Intestinal type & 6 & 10.7 \\
\hline Barrett & 4 & 7.1 \\
\hline Signet ring cell & 7 & 12.5 \\
\hline HER-2 positive & 3 & 5.4 \\
\hline \multicolumn{3}{|l|}{ Location } \\
\hline AEG I-III & 30 & 53.6 \\
\hline Gastric & 26 & 46.4 \\
\hline \multicolumn{3}{|l|}{ Number of metastases } \\
\hline 1 & 35 & 62.5 \\
\hline 2 & 21 & 37.5 \\
\hline \multicolumn{3}{|l|}{ Line ramucirumab $\pm \mathrm{CHT}$} \\
\hline Second-line & 46 & 82.1 \\
\hline$>$ Second-line & 10 & 17.9 \\
\hline \multicolumn{3}{|l|}{ Ramucirumab $\mathrm{CHT}$ regimen } \\
\hline Paclitaxel & 38 & 67.9 \\
\hline FOLFIRI & 16 & 28.6 \\
\hline Monotherapy/other & 2 & 3.6 \\
\hline \multicolumn{3}{|c|}{ Prior neoadjuvant treatment $(n=29)(52 \%)$} \\
\hline Cisplatin/5-FU & 4 & 13.8 \\
\hline FLOT & 12 & 41.4 \\
\hline Other & 13 & 44.8 \\
\hline \multicolumn{3}{|c|}{$\begin{array}{l}\text { First-line palliative/neoadjuvant treatment prior } \\
\text { to ramucirumab combined with } \mathrm{CHT}(\mathrm{n}=56)\end{array}$} \\
\hline FLOT (perioperative) & 12 & 21.4 \\
\hline Cis- or carboplatin/5-FU & 13 & 23.2 \\
\hline FOLFOX & 7 & 12.5 \\
\hline DCF & 4 & 7.1 \\
\hline $\mathrm{CHT} \pm$ trastuzumab & 3 & 5.4 \\
\hline Other & 17 & 30.4 \\
\hline
\end{tabular}

$\mathrm{CHT}$, chemotherapy.
Table 2 Outcomes and response

\begin{tabular}{|c|c|c|}
\hline Variable & Months (median, 95\% Cl) & $P$ \\
\hline \multicolumn{3}{|c|}{$\begin{array}{l}\text { Ramucirumab + CHT (overall) } \\
(n=56)\end{array}$} \\
\hline PFS & $3.0(2.2-3.8)$ & \\
\hline Second-line & $3.3(2.3-4.4)$ & \\
\hline$>$ Second-line & $2.9(1.9-3.8)$ & 0.7 \\
\hline OS & $5.0(2.9-7.0)$ & \\
\hline Second-line & $5.0(1.2-8.7)$ & 0.79 \\
\hline$>$ Second-line & $4.3(0.4-8.3)$ & \\
\hline \multicolumn{3}{|l|}{ ORR $(\%)(n=47)$} \\
\hline PR & 12.8 & \\
\hline SD & 48.9 & \\
\hline PD & 38.3 & \\
\hline \multicolumn{3}{|c|}{$\begin{array}{l}\text { Ramucirumab + paclitaxel } \\
(\mathrm{n}=38)\end{array}$} \\
\hline PFS & $2.9(2.3-3.6)$ & \\
\hline os & $4.4(4.1-4.7)$ & \\
\hline \multicolumn{3}{|l|}{ ORR $(\%)(n=32)$} \\
\hline PR & 9.4 & \\
\hline SD & 53.1 & \\
\hline PD & 37.5 & \\
\hline \multicolumn{3}{|c|}{ Ramucirumab + FOLFIRI $(n=16)$} \\
\hline PFS & $5.9(0.35-11.4)$ & \\
\hline OS & $8.3(6.6-10)$ & \\
\hline \multicolumn{3}{|l|}{ ORR (\%) $(n=13)$} \\
\hline PR & 23.1 & \\
\hline SD & 46.1 & \\
\hline PD & 30.8 & \\
\hline
\end{tabular}

and $12.8 \%$ had a partial response, translating into a clinical benefit rate of $61.7 \%$ (Figure 1, Table 2).

Ramucirumab was given in combination with paclitaxel in 38 patients, translating into a median PFS and OS of 2.9 (95\% CI: $2.3-3.6)$ and 4.4 (95\% CI: 4.1-4.7) months, respectively. 


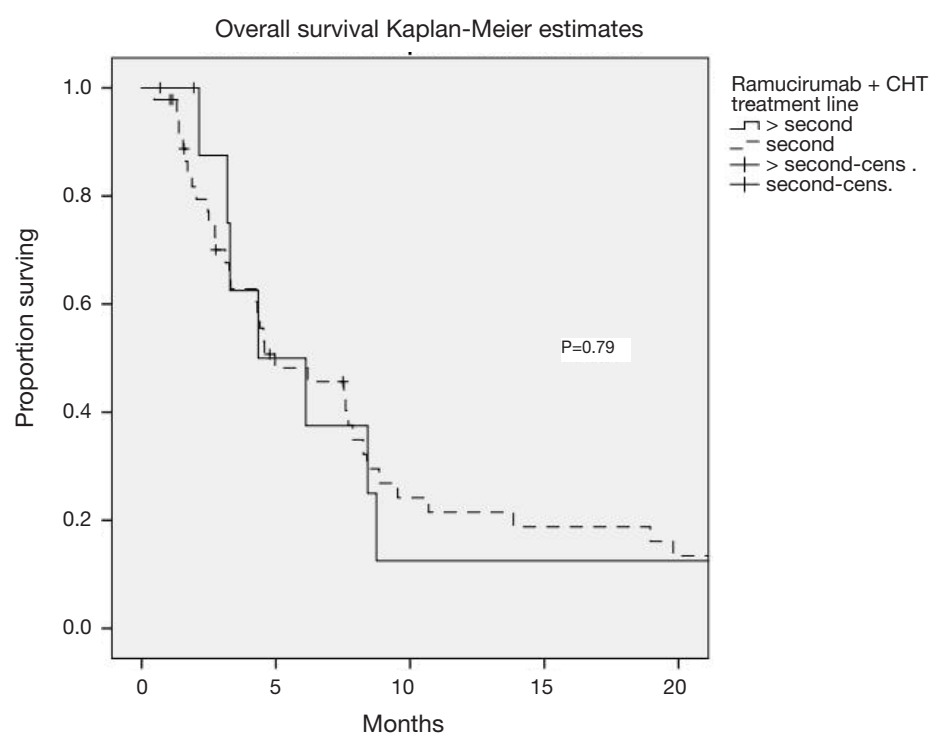

Figure 1 OS of ramucirumab + CHT in second- and beyond second-line palliative treatment (n=56). OS, overall survival; CHT, chemotherapy.

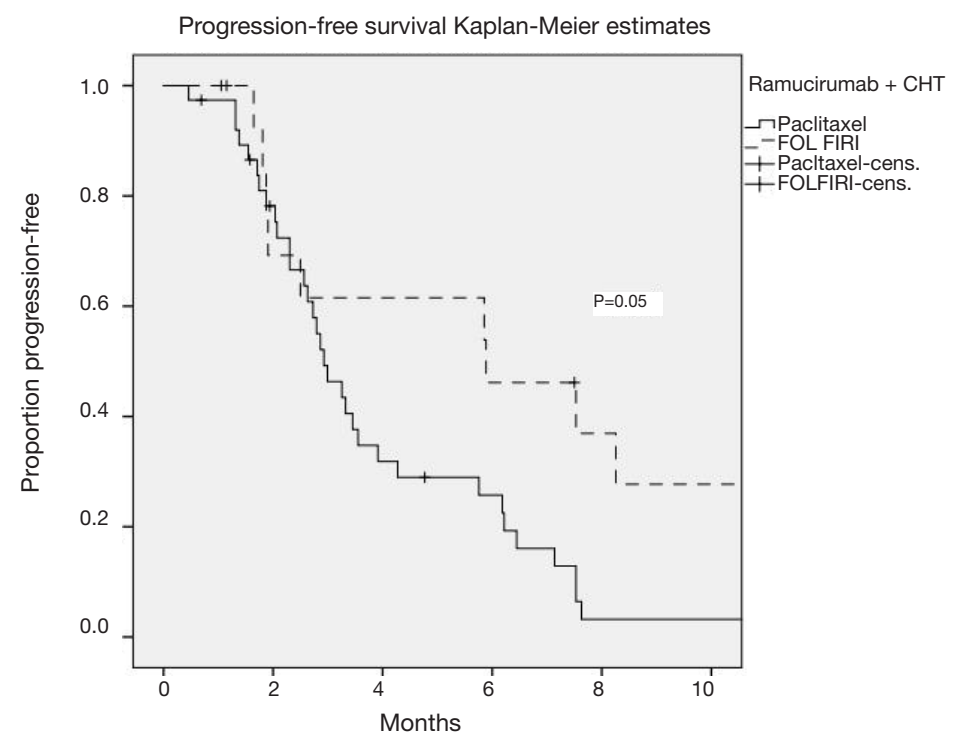

Figure 2 PFS FOLFIRI-R (n=16) and pacl-R (n=38). PFS, progression-free survival.

Sixteen patients were not considered eligible for treatment containing paclitaxel, mostly because of highgrade PNP or a short interval between perioperative taxane-containing CHT and a short interval of relapse. In these patients, FOLFIRI was used as CHT backbone in combination with ramucirumab. The median PFS and OS were 5.9 (95\% CI: 5.5-6.5) and 8.3 (6.6-10) months, respectively. The ORR was $23 \%$, with only $31 \%$ of patients having progressed at first response assessment.
The median PFS was significantly better in FOLFIRI$\mathrm{R}$-treated patients than in paclitaxel-R-treated patients $(\mathrm{P}=0.05)$, independent of the treatment line (Figure 2).

\section{Outcome of patients perioperatively treated with FLOT}

A total of 12 patients received the taxane-based FLOT regimen as initial perioperative treatment. Among them 5 patients received paclitaxel, and 7 patients received 


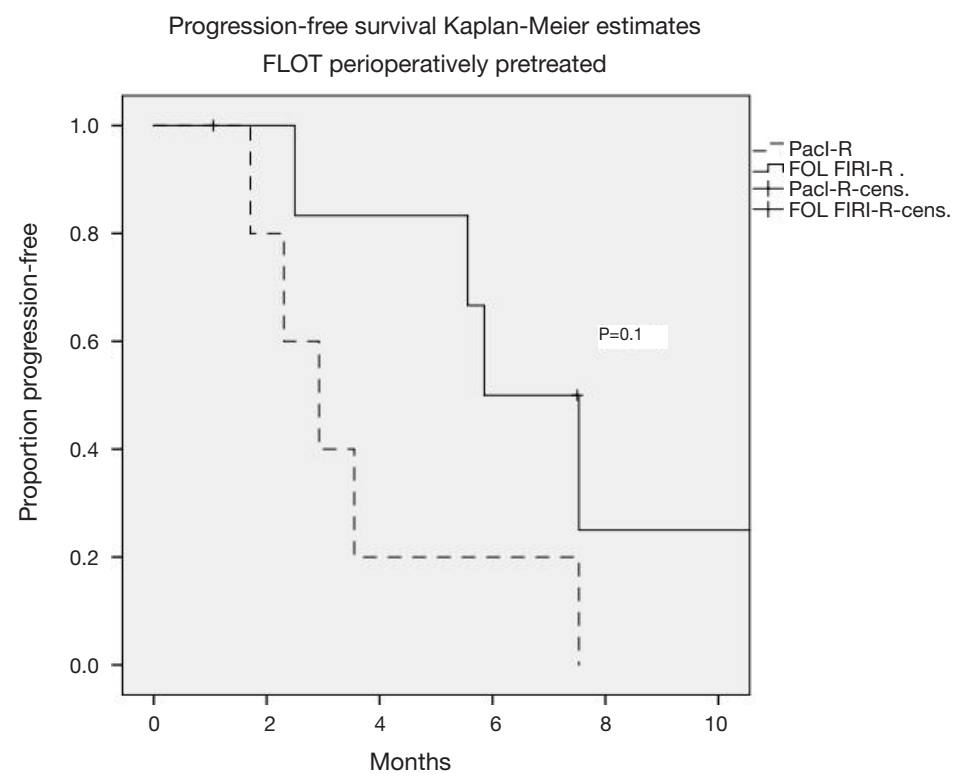

Figure 3 PFS of patients after perioperative FLOT $(\mathrm{n}=12)(\mathrm{P}=0.1)$. PFS, progression-free survival.

FOLFIRI in combination with ramucirumab. The median PFS was 2.9 (95\% CI: 1.6-4.3) months for paclitaxel-R and 5.6 (95\% CI: 4-7.8) months for FOLFIRI-R $(\mathrm{P}=0.1)$ in this small subgroup of patients (Figure 3).

\section{Toxicity}

Overall toxicity was manageable and consistent with that reported in published phase III trials $(11,12)$. The most common grade 3 toxicity for patients treated with paclitaxel-R and FOLFIRI-R was neutropenia (19\% vs. $44 \%$ ), followed by diarrhoea, fatigue and PNP. FOLFIRI-R toxicity rates were generally high and led to dose reductions of $69 \%$ of patients. Only $22 \%$ of patients receiving paclitaxel-R were dose reduced. Adverse events potentially associated with the VEGF pathway were uncommon, with approximately $7 \%$ of patients developing grade 1 or 2 hypertension, but no bleeding or thromboembolic events of higher grade were documented. The rates of proteinuria were not well captured in this retrospective cohort. However, no reported cases of nephrotic syndrome were observed.

\section{Discussion}

Based on phase III data, paclitaxel-R constitutes a common second-line strategy for HER-2-negative disease (11). Other available options include administration of the single agent ramucirumab (12), irinotecan (24,25), docetaxel (26) or paclitaxel (27). Ramucirumab monotherapy is feasible, especially in frail patients, although ECOG 2 patients were not included in the REGARD trial (12). In our patient series, only two patients were treated with ramucirumab monotherapy because of frailty and taxane pretreatment.

Our data revealed a median PFS and OS of only three and five months, respectively, in the overall population independent of the CHT backbone and treatment line. In the pivotal phase III RAINBOW study, the median PFS was substantially longer than that in our patient series. One possible reason for this difference could be the patient characteristics in our population. Almost $50 \%$ of our patients presented with peritoneal metastases. This is known to be an adverse prognostic factor in gastric cancer and in treatment with ramucirumab (12). Real-world data from the RAMOSS trial showed that the presence of peritoneal metastases was associated with a significantly worse outcome (HR 1.57) (28,29). Moreover, more than two-thirds of patients presented with the diffuse infiltrating subtype, which responds poorly to CHT (30). Interestingly, half of patients had tumours of the GEJ, which are associated with a dismal prognosis, and 5-year survival rates in the range of $14 \%$ to $22 \%(5,31)$. Although most of our patients had a good performance status, these characteristics could influence the outcome in our real-world population. Another characteristic that differentiates clinical trial results from real-world data is the definition of progressive disease 
and a switch in systemic treatment.

In daily clinical practice, progressive disease is also defined by clinical progression, which is an important parameter in daily practice but not decisive in clinical trials. Peritoneal carcinomatosis is often not a measurable target lesion, and radiographic progression is difficult to prove. Therefore, some patients would have experienced longer PFS if only radiographic progression was taken into account. The literature shows a number of real-world data that also report short PFS and OS, as in published phase III trials (28,32-34). Nowadays with the emerging development of immunoncology drugs, clinical progression is a valid parameter to define progression and end of treatment in these trials. A recent metaanalysis of targeted anticancer therapies showed that the drugs had a greater effect on PFS than OS, but that PFS benefits often did not translate to OS benefits (35).

In daily practice, contraindications against ramucirumab are rarely present. A recent thromboembolic event, instable cardiovascular disease, uncontrolled hypertension or wound healing disorders might be contraindications for ramucirumab application. Paclitaxel, which is the only drug that has been published to be efficient in combination with ramucirumab in gastric cancer as a second-line treatment, is often contraindicated because of pretreatment-associated conditions. Taxanes should not be used in patients with neuropathy grade $>1$. Patients who are perioperatively treated with FLOT or DCF often suffer from residual PNP. Moreover, if relapse occurs within a short period after a taxane-based perioperative treatment protocol, resistance against paclitaxel can be assumed.

An alternative taxane-free regimen for platinum- and 5-FU-pretreated patients is irinotecan as monotherapy (24), with a median PFS of 4 months, or FOLFIRI, which has proven evidence in the first-line treatment of advanced or metastatic gastric cancer (median PFS: 4.3 months) (36). Our data show encouraging responses and prolonged PFS and OS when FOLFIRI is combined with ramucirumab in this setting, suggesting a potential synergistic effect of these drugs.

We treated 16 patients who were ineligible for taxanebased treatment or had been pretreated with FLOT perioperatively $(\mathrm{n}=12)$ with FOLFIRI-R. The median PFS of patients treated with FOLFIRI-R was significantly longer than in paclitaxel-R-treated patients (5.9 months), with an ORR of $23 \%$. A phase II trial (RAMIRIS) is investigating this treatment for 5-FU and platinum- or taxane-pretreated patients. Reports from phase Ib were already promising (37), this year the first interim analysis of safety and efficacy of this combination in 58 patients including taxane pre-treated patient has been reported (20). Toxicity was documented as expected from the approved regimen in colorectal cancer. Surprisingly haematotoxicity was lower (grade 3 neutropenia 20\% in der FOLFIRI-R) than the paclitaxel-R group in the RAMIRIS trial. Published data from colorectal cancer trials, investigating FOLFIRI-R have shown grade 3 neutropenia up to $38 \%$. We reported a higher rate of grade 3 neutropenia and dose reduction compared to paclitaxel-R but no cases of neutropenic fever. These results are also consistent with published data from a multi-institutional retrospective analysis with 29 patients receiving FOLFIRI-R as $2 \mathrm{~L}$ treatment. The median PFS was 6 months, and the ORR was $23 \%$. No new safety signals were reported (19).

We further report data for the subgroup of FLOTpretreated patients that switched to a non-taxane-based FOLFIRI CHT backbone for ramucirumab that holds promise as an effective palliative treatment regimen. Our data included a similar subgroup of patients that have been pre-treated with docetaxel as reported in the phase II RAMIRIS trial interim analysis (20). The abstract reports 29 docetaxel pre-treated patients with an ORR of $30 \%$ in the FOLFIRI-R arm and 50\% in the paclitaxel-R arm. So far, no PFS or OS results have been reported from the RAMIRIS trial. This report is consistent with our data that showed a trend towards prolonged PFS in patients treated with FOLFIRI-R compared to paclitaxel-R in this pretreated cohort. Limitations are given due to the small patient number, but these findings might encourage further investigations in this specific patient cohort. To the best of our knowledge, this is the first efficacy report including PFS results of FOLFIRI-R after relapse on FLOT treatment.

\section{Conclusions}

Metastatic gastric or GEJ cancer remains a disease that is difficult to treat. In taxane-pretreated patients or those with persistent PNP, the combination of FOLFIRI-R might represent a promising combination.

\section{Acknowledgments}

We thank Madeleine Blum for constantly updating the patient data base.

Funding: None.

\section{Footnote}

Conflicts of Interest: All authors have completed the ICMJE 
uniform disclosure form (available at http://dx.doi. org/10.21037/jgo.2020.03.10). The authors have no conflicts of interest to declare.

Ethical Statement: The authors are accountable for all aspects of the work in ensuring that questions related to the accuracy or integrity of any part of the work are appropriately investigated and resolved. Ethics approval was not necessary for this retrospective analysis. All patients of our clinic sign a declaration form that their data can be used for research purposes.

Open Access Statement: This is an Open Access article distributed in accordance with the Creative Commons Attribution-NonCommercial-NoDerivs 4.0 International License (CC BY-NC-ND 4.0), which permits the noncommercial replication and distribution of the article with the strict proviso that no changes or edits are made and the original work is properly cited (including links to both the formal publication through the relevant DOI and the license). See: https://creativecommons.org/licenses/by-ncnd/4.0\%.

\section{References}

1. SEER Cancer Statistics Review, 1975-2014, National Cancer Institute. Bethesda, MD [database on the Internet]2015. Available online: https://seer.cancer.gov/ csr/1975_2016

2. Kamangar F, Dores GM, Anderson WF. Patterns of cancer incidence, mortality, and prevalence across five continents: defining priorities to reduce cancer disparities in different geographic regions of the world. J Clin Oncol 2006;24:2137-50.

3. Blaser MJ, Chen Y. A New Gastric Cancer Among Us. J Natl Cancer Inst 2018;110:549-50.

4. Al-Batran SE, Homann N, Pauligk C, et al. Perioperative chemotherapy with fluorouracil plus leucovorin, oxaliplatin, and docetaxel versus fluorouracil or capecitabine plus cisplatin and epirubicin for locally advanced, resectable gastric or gastro-oesophageal junction adenocarcinoma (FLOT4): a randomised, phase 2/3 trial. Lancet 2019;393:1948-57.

5. Cunningham D, Allum WH, Stenning SP, et al. Perioperative chemotherapy versus surgery alone for resectable gastroesophageal cancer. $\mathrm{N}$ Engl J Med 2006;355:11-20.

6. Maeda K, Chung YS, Ogawa Y, et al. Prognostic value of vascular endothelial growth factor expression in gastric carcinoma. Cancer 1996;77:858-63.

7. Ohtsu A, Shah MA, Van Cutsem E, et al. Bevacizumab in combination with chemotherapy as first-line therapy in advanced gastric cancer: a randomized, doubleblind, placebo-controlled phase III study. J Clin Oncol 2011;29:3968-76.

8. Cunningham D, Stenning SP, Smyth EC, et al. Perioperative chemotherapy with or without bevacizumab in operable oesophagogastric adenocarcinoma (UK Medical Research Council ST03): primary analysis results of a multicentre, open-label, randomised phase 2-3 trial. Lancet Oncol 2017;18:357-70.

9. Shah MA, Jhawer M, Ilson DH, et al. Phase II study of modified docetaxel, cisplatin, and fluorouracil with bevacizumab in patients with metastatic gastroesophageal adenocarcinoma. J Clin Oncol 2011;29:868-74.

10. Shen L, Li J, Xu J, et al. Bevacizumab plus capecitabine and cisplatin in Chinese patients with inoperable locally advanced or metastatic gastric or gastroesophageal junction cancer: randomized, double-blind, phase III study (AVATAR study). Gastric Cancer 2015;18:168-76.

11. Wilke H, Muro K, Van Cutsem E, et al. Ramucirumab plus paclitaxel versus placebo plus paclitaxel in patients with previously treated advanced gastric or gastro-oesophageal junction adenocarcinoma (RAINBOW): a double-blind, randomised phase 3 trial. Lancet Oncol 2014;15:1224-35.

12. Fuchs CS, Tomasek J, Yong CJ, et al. Ramucirumab monotherapy for previously treated advanced gastric or gastro-oesophageal junction adenocarcinoma (REGARD): an international, randomised, multicentre, placebocontrolled, phase 3 trial. Lancet 2014;383:31-9.

13. Liu W, Dong Z, Hu R, et al. Association of Vascular Endothelial Growth Factor ( VEGF) Gene Polymorphisms With Gastric Cancer and Its Development, Prognosis, and Survival. Technol Cancer Res Treat 2018;17:1533034617753810.

14. Pavlakis N, Sjoquist KM, Martin AJ, et al. Regorafenib for the Treatment of Advanced Gastric Cancer (INTEGRATE): A Multinational Placebo-Controlled Phase II Trial. J Clin Oncol 2016;34:2728-35.

15. Moehler M, Gepfner-Tuma I, Maderer A, et al. Sunitinib added to FOLFIRI versus FOLFIRI in patients with chemorefractory advanced adenocarcinoma of the stomach or lower esophagus: a randomized, placebo-controlled phase II AIO trial with serum biomarker program. BMC Cancer 2016;16:699.

16. Moehler M, Mueller A, Hartmann JT, et al. An open- 
label, multicentre biomarker-oriented AIO phase II trial of sunitinib for patients with chemo-refractory advanced gastric cancer. Eur J Cancer 2011;47:1511-20.

17. Li J, Qin S, Xu J, et al. Randomized, Double-Blind, Placebo-Controlled Phase III Trial of Apatinib in Patients With Chemotherapy-Refractory Advanced or Metastatic Adenocarcinoma of the Stomach or Gastroesophageal Junction. J Clin Oncol 2016;34:1448-54.

18. Shitara K, Kadowaki S, Nishina T, et al. Safety, pharmacokinetic, and clinical activity profiles of ramucirumab in combination with three platinum/ fluoropyrimidine doublets in Japanese patients with chemotherapy-naive metastatic gastric/gastroesophageal junction cancer. Gastric Cancer 2018;21:106-13.

19. Klempner SJ, Maron SB, Chase L, et al. Initial Report of Second-Line FOLFIRI in Combination with Ramucirumab in Advanced Gastroesophageal Adenocarcinomas: A Multi-Institutional Retrospective Analysis. Oncologist 2019;24:475-82.

20. Lorenzen S, Thuss-Patience PC, Pauligk C, et al. FOLFIRI plus ramucirumab versus paclitaxel plus ramucirumab for patients with advanced or metastatic adenocarcinoma of the stomach or gastroesophageal junction as second-line therapy: Interim safety and efficacy results from the phase II RAMIRIS Study (AIOSTO-0415) of the German Gastric Group at AIO. J Clin Oncol 2019;37:4023.

21. Tabernero J, Yoshino T, Cohn AL, et al. Ramucirumab versus placebo in combination with second-line FOLFIRI in patients with metastatic colorectal carcinoma that progressed during or after first-line therapy with bevacizumab, oxaliplatin, and a fluoropyrimidine (RAISE): a randomised, double-blind, multicentre, phase 3 study. Lancet Oncol 2015;16:499-508.

22. Eisenhauer EA, Therasse P, Bogaerts J, et al. New response evaluation criteria in solid tumours: revised RECIST guideline (version 1.1). Eur J Cancer 2009;45:228-47.

23. Bang YJ, Van Cutsem E, Feyereislova A, et al. Trastuzumab in combination with chemotherapy versus chemotherapy alone for treatment of HER2-positive advanced gastric or gastro-oesophageal junction cancer (ToGA): a phase 3, open-label, randomised controlled trial. Lancet 2010;376:687-97.

24. Thuss-Patience PC, Kretzschmar A, Bichev D, et al. Survival advantage for irinotecan versus best supportive care as second-line chemotherapy in gastric cancer--a randomised phase III study of the Arbeitsgemeinschaft
Internistische Onkologie (AIO). Eur J Cancer 2011;47:2306-14.

25. Hironaka S, Ueda S, Yasui H, et al. Randomized, openlabel, phase III study comparing irinotecan with paclitaxel in patients with advanced gastric cancer without severe peritoneal metastasis after failure of prior combination chemotherapy using fluoropyrimidine plus platinum: WJOG 4007 trial. J Clin Oncol 2013;31:4438-44.

26. Ford HE, Marshall A, Bridgewater JA, et al. Docetaxel versus active symptom control for refractory oesophagogastric adenocarcinoma (COUGAR-02): an open-label, phase 3 randomised controlled trial. Lancet Oncol 2014;15:78-86.

27. Kang JH, Lee SI, Lim DH, et al. Salvage chemotherapy for pretreated gastric cancer: a randomized phase III trial comparing chemotherapy plus best supportive care with best supportive care alone. J Clin Oncol 2012;30:1513-8.

28. Di Bartolomeo M, Niger M, Tirino G, et al. Ramucirumab as Second-Line Therapy in Metastatic Gastric Cancer: Real-World Data from the RAMoss Study. Target Oncol 2018;13:227-34.

29. Fuchs CS, Muro K, Tomasek J, et al. Prognostic Factor Analysis of Overall Survival in Gastric Cancer from Two Phase III Studies of Second-line Ramucirumab (REGARD and RAINBOW) Using Pooled Patient Data. J Gastric Cancer 2017;17:132-44.

30. Maeda H, Okabayashi T, Nishimori I, et al. Clinicopathologic features of adenocarcinoma at the gastric cardia: is it different from distal cancer of the stomach? J Am Coll Surg 2008;206:306-10.

31. DeMeester SR. Adenocarcinoma of the esophagus and cardia: a review of the disease and its treatment. Ann Surg Oncol 2006;13:12-30.

32. Paulson AS, Hess LM, Liepa AM, et al. Ramucirumab for the treatment of patients with gastric or gastroesophageal junction cancer in community oncology practices. Gastric Cancer 2018;21:831-44.

33. Jung M, Ryu MH, Oh DY, et al. Efficacy and tolerability of ramucirumab monotherapy or in combination with paclitaxel in gastric cancer patients from the Expanded Access Program Cohort by the Korean Cancer Study Group (KCSG). Gastric Cancer 2018;21:819-30.

34. Yamaguchi K, Fujitani K, Nagashima F, et al. Ramucirumab for the treatment of metastatic gastric or gastroesophageal junction adenocarcinoma following disease progression on first-line platinum- or fluoropyrimidine-containing combination therapy in Japanese patients: a phase 2, open- 
label study. Gastric Cancer 2018;21:1041-9.

35. Tan A, Porcher R, Crequit P, et al. Differences in Treatment Effect Size Between Overall Survival and Progression-Free Survival in Immunotherapy Trials: A Meta-Epidemiologic Study of Trials With Results Posted at ClinicalTrials.gov. J Clin Oncol 2017;35:1686-94.

36. Guimbaud R, Louvet C, Ries P, et al. Prospective, randomized, multicenter, phase III study of fluorouracil, leucovorin, and irinotecan versus epirubicin, cisplatin, and capecitabine in advanced gastric adenocarcinoma:

Cite this article as: Vogl UM, Vormittag L, Winkler T, Kafka A, Weiser-Jasch O, Heinrich B, Roider-Schur S, Andalibi H, Autzinger E, Schima W, Klaus A, Zacherl J, Wimberger GM, Öhler L. Ramucirumab plus paclitaxel or FOLFIRI in platinum-refractory advanced or metastatic gastric or gastroesophageal junction adenocarcinoma-experience at two centres. J Gastrointest Oncol 2020;11(2):366-375. doi: 10.21037/jgo.2020.03.10 a French intergroup (Federation Francophone de Cancerologie Digestive, Federation Nationale des Centres de Lutte Contre le Cancer, and Groupe Cooperateur Multidisciplinaire en Oncologie) study. J Clin Oncol 2014;32:3520-6.

37. Satake H, Sagawa T, Fujikawa K, et al. Phase Ib study of irinotecan and ramucirumab for advanced gastric cancer previously treated with fluoropyrimidine with/without platinum and taxane. Cancer Chemother Pharmacol 2018;82:839-45. 